\title{
Progressive early passive and active exercise therapy after surgical rotator cuff repair - study protocol for a randomized controlled trial (the CUT-N-MOVE trial)
}

Birgitte Hougs Kjær ${ }^{1,2^{*}}$ (D, S. Peter Magnusson ${ }^{1,3}$, Susan Warming ${ }^{1}$, Marius Henriksen ${ }^{1,5}$, Michael Rindom Krogsgaard ${ }^{4}$ and Birgit Juul-Kristensen ${ }^{2}$

\begin{abstract}
Background: Rotator cuff tear is a common cause of shoulder disability and results in patients predominantly complaining of pain and loss of motion and strength. Traumatic rotator cuff tears are typically managed surgically followed by $\sim 20$ weeks of rehabilitation. However, the timing and intensity of the postoperative rehabilitation strategy required to reach an optimal clinical outcome is unknown. Early controlled and gradually increased tendon loading has been suggested to positively influence tendon healing and recovery. The aim of this trial is therefore to examine the effect of a progressive rehabilitation strategy on pain, physical function and quality of life compared to usual care (that limits tendon loading in the early postoperative phase) in patients who have a rotator cuff repair of a traumatic tear.
\end{abstract}

Methods: The current study is a randomized, controlled, outcome-assessor blinded, multicenter, superiority trial with a two-group paralleled design. A total of 100 patients with surgically repaired traumatic rotator cuff tears will be recruited from up to three orthopedic departments in Denmark, and randomized to either a progressive early passive and active movement program or a limited early passive movement program (usual care). The primary outcome measure will be the change from pre-surgery to 12 weeks post-surgery in the Western Ontario Rotator Cuff Index questionnaire. Secondary outcomes include the Disabilities Arm, Shoulder and Hand questionnaire $(\mathrm{DASH})$, range of motion, strength and tendon healing characteristics from ultrasound measurements at 12 months follow up.

Discussion: We hypothesized that patients who receive the progressive rehabilitation strategy will benefit more with respect to pain reduction, physical function and quality of life than those who receive care as usual. If this is confirmed our study can be used clinically to enhance the recovery of patients with traumatic rotator cuff tear.

Trial registration: ClinicalTrials.gov, NCT02969135. Registered on 15 November 2016.

Keywords: Shoulder, Rotator cuff, Tear, Rupture, Physiotherapy, Rehabilitation, Exercise therapy

\footnotetext{
* Correspondence: birgitte.hougs.kjaer@regionh.dk

${ }^{1}$ Department of Physical and Occupational Therapy, Bispebjerg-Frederiksberg

Hospital, Bispebjerg Bakke 23, DK-2400 Copenhagen, Denmark

${ }^{2}$ Department of Sports Science and Clinical Biomechanics, University of

Southern Denmark, Campusvej 55, DK-5230 Odense, Denmark

Full list of author information is available at the end of the article
}

(c) The Author(s). 2018 Open Access This article is distributed under the terms of the Creative Commons Attribution 4.0 International License (http://creativecommons.org/licenses/by/4.0/), which permits unrestricted use, distribution, and reproduction in any medium, provided you give appropriate credit to the original author(s) and the source, provide a link to the Creative Commons license, and indicate if changes were made. The Creative Commons Public Domain Dedication waiver (http://creativecommons.org/publicdomain/zero/1.0/) applies to the data made available in this article, unless otherwise stated. 


\section{Background}

Shoulder pain is the third most common musculoskeletal disorder with a lifetime prevalence of $30 \%[1,2]$. Shoulder disorders are often persistent and recurrent, and 54\% of patients report some symptoms after 3 years [1,3]. Moreover, it often impacts people's daily life and work capacity dramatically [4-6]. Rotator cuff tears, which can be due to trauma or degeneration [7], are common causes of chronic shoulder pain and disability, especially with advancing age $[1,3,8,9]$. The predominant shoulder complaints among patients are pain, loss of motion and strength during arm elevation, which results in loss of function $[3,9]$. One of the criteria for surgery is symptomatic full-thickness tears, or partial-thickness tears that are symptomatically resistant to non-surgical intervention $[10,11]$. The incidence of rotator cuff surgery, based on surgery for both non-traumatic and traumatic tears, is increasing worldwide $[12,13]$, and The National Patient Register in Denmark recorded 730 rotator cuff repairs in 2006 and 990 in 2012, which represents a $35 \%$ increase [14].

The Danish National Clinical Guideline on selected shoulder disorders recommends that these patients are offered rehabilitation after surgical repair, and that the shoulder is immobilized post-surgery (usual care) [14]. However, the existing clinical studies of postoperative treatment targeting patients with rotator cuff repair are of moderate to low quality (lack of blinding, small sample sizes, lack/poor responsiveness on outcomes, and/or with only per protocol analyses) [15-20], making it difficult to unequivocally conclude what treatment regimen is the best. Studies on the timing of rehabilitation have showed a smaller positive short-term effect of early active rehabilitation (from postoperative day 21) compared to later rehabilitation [15], but no superior effect of early passive exercises $[17,19,20]$. A small or no effect was seen in studies focusing on the intensity of rehabilitation, including passive exercises versus immobilization [16] and progressive versus limited early passive exercises [18]. Recent systematic reviews on the effect of rehabilitation after rotator cuff surgery confirms that early range of motion (ROM) exercises accelerate healing, reduce stiffness, do not increase the risk of re-tear and that immobilization does not increase tendon healing or the clinical outcome [21-24]. However, high-quality and adequately powered trials with standardized treatment protocols testing early initiation of rehabilitation and gradual introduction of functional load and including important clinical outcomes such as return to work are needed $[25,26]$.

Research on rotator cuff tears often focuses on improvement of surgery techniques [9, 27-31], biomechanics and biology of tendon healing [16, 32, 33]; however, little is known about the effects of postoperative rehabilitation. Human autopsy studies indicate that the tendon can regain its ability to transmit almost the same amount of force as an intact tendon if it is fully repaired [10, 33]. The magnitude of retraction will markedly influence the rotator cuff muscle generating capacity [33]. Based on autopsy and physiological data [34] it has been suggested that rehabilitation should include reestablishment of joint ROM, shoulder function and muscular strength while considering the healing time of the repaired tendon.

The metabolic turnover of tendon tissue is slower than in muscle [35-37], and therefore a controlled and gradual increase in tendon loading has been suggested to achieve optimal tendon healing $[38,39]$. This includes starting rehabilitation in the proliferative phase of healing and continuing into the remodeling phase so that the collagen is subjected to a load that is beneficial for the formation and the final strength of the tendon [35]. This means that postoperative immobilization may decrease tendon strength [40-42], and therefore increase the risk of a re-tear $[34,43,44]$. Further, immobilization may also result in adhesions and decreased ROM [38, 45]. Consequently, early passive motion postoperatively has gained acceptance [35, 38, 46].

In summary, no high-quality study has evaluated the combined effect of early (= the time of initiation) and progressive (= with an increased intensity, also including active muscle contraction) postoperative exercises on physical function, pain, quality of life and biological tendon healing. Therefore, the short-term (12 weeks) and long-term (12 months) effects of an early and targeted progressive postoperative exercise therapy program remain to be investigated. The primary aim of this trial is to evaluate the effect of a 12-week progressive rehabilitation (PR) strategy on shoulder function compared to usual care (UC) in patients recovering from surgical treatment of rotator cuff tears. The secondary aims include the effects on shoulder ROM, muscle strength, return to work rates and tendon healing characteristics. Finally, a prediction of health outcomes will be performed in both groups.

\section{Methods/design}

This is a randomized, controlled, outcome-assessor blinded, superiority trial, called CUT-N-MOVE with a two-group parallel design comparing a progressive rehabilitation (PR) strategy with usual care (UC). It is a multicenter study with two phases; the first phase includes the main trial with a baseline and a 12-week follow-up, which corresponds to the planned duration of the experimental individualized rehabilitation program (Fig. 1). The primary endpoint is 12 weeks from the baseline.

The second phase includes the secondary endpoint 52 weeks after baseline, at which point Ultrasound (US) measurements are included in the outcome assessments. During the 40-week follow up period (from week 12 to week 52) most patients continue standard rehabilitation in the community for 4-8 weeks. The community care providers 


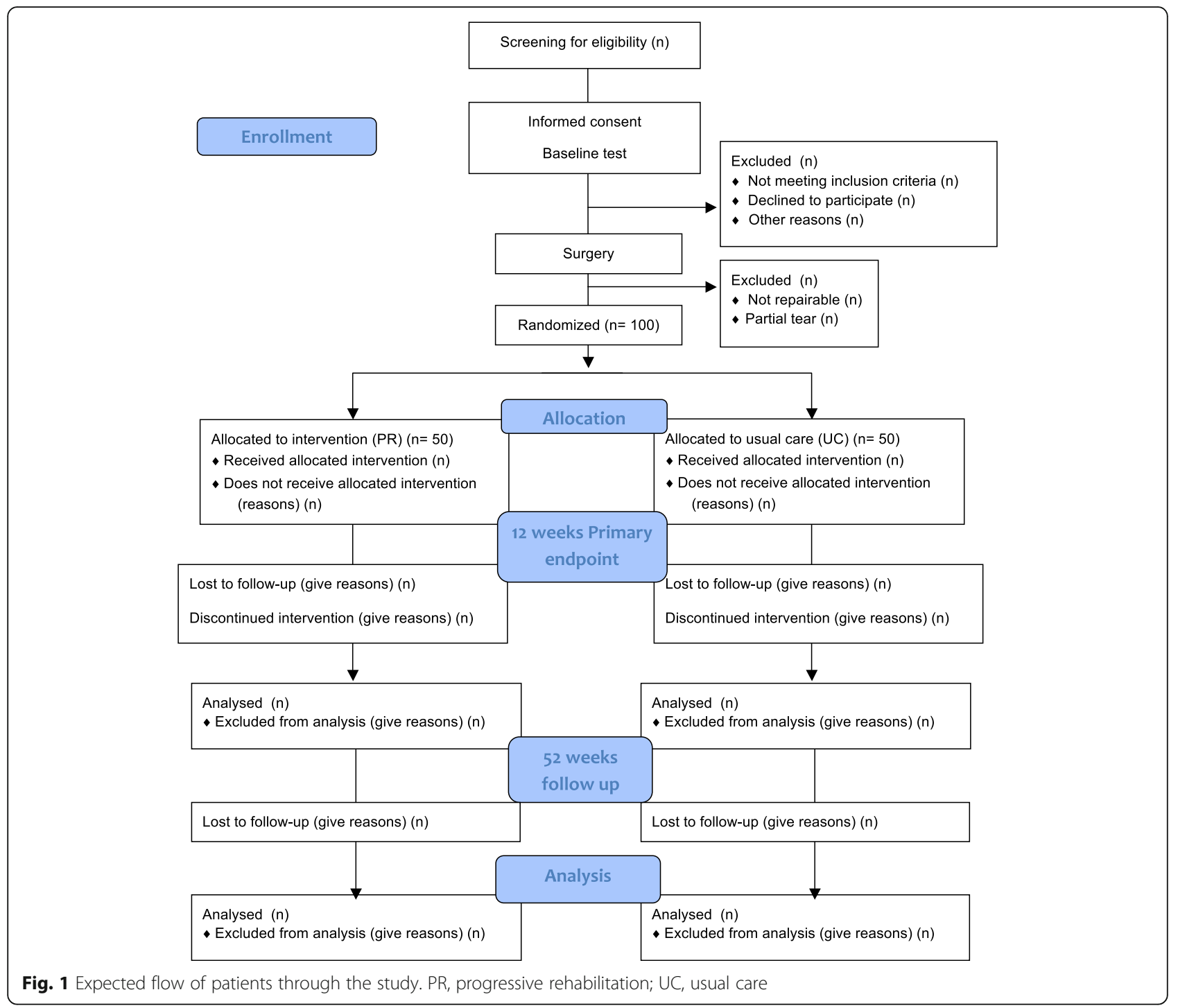

are not informed about initial treatment to which each patient has been allocated (unless the patients tell them) and can thus be considered quasi-blinded.

After 12 weeks no study-related activities are performed, except for the 12-month follow up and treatment of any adverse events that might occur during the trial (52 weeks). Patients are randomized equally (1:1) to receive either PR or UC (Fig. 1).

\section{Patients and settings}

Patients are recruited from the Section for Sports Traumatology, Department of Orthopedic Surgery, Bispebjerg-Frederiksberg Hospital and from The Shoulder-Elbow Unit, Herlev-Gentofte Hospital, both in Denmark. Additional inclusion sites may be added.
Patients may be enrolled in the study provided they meet all of the inclusion criteria:

- Age minimum 18 years

- A clinical diagnosis of traumatic full-thickness rotator cuff (RC) tear involving the supraspinatus tendon (full thickness and width) verified by arthroscopy

- A repairable tear

In trauma the trauma mechanism should be described. Typically it will be a forced abduction and external rotation to mitigate for a fall, a fall on the outstretched arm, a pull in the arm or a shoulder luxation. No previous symptoms are implied within the definition of a traumatic tear.

Patients are excluded if they fulfill any of the following exclusion criteria: 
1. A non-traumatic $\mathrm{RC}$ tear of the shoulder

2. An isolated teres minor or subscapularis tear

3. A partial-thickness/partial-width tear

4. Prior shoulder surgery (all shoulder joints)

5. A clinical diagnosis of glenohumeral osteoarthritis (OA), rheumatoid arthritis or periarthrosis

6. Unable to speak or read Danish

7. Unable to perform and maintain the exercise therapy

8. Other conditions that negatively influence compliance, or place the patient at increased risk, or otherwise make them unsuitable for participation

Orthopedic surgeons perform an initial screening of all patients referred to the department for surgery. If the patients fulfill the eligibility criteria they are referred to the principal investigator, who performs the final eligibility assessment, give the patient detailed information about the study and ask for consent to participate in the study. If the patient consents to participate a baseline assessment visit is performed between 1 and 14 days before surgery, on the understanding that the patient is not finally included and allocated until the surgeon verifies a total and repairable supraspinatus tear. After surgery the orthopedic surgeon provides surgery information (verifying reparable tendon (s)), and included patients are electronically randomized (described in "Allocation, randomization and sequence generation").

The rehabilitation sessions are conducted at the local physiotherapy departments by 10 orthopedic trained physiotherapists with postgraduate musculoskeletal experience between 8 and 25 years, who have been thoroughly trained in the rehabilitation protocols, including the different phases, restrictions and progression.

\section{Interventions}

Patients in both treatment groups have the affected shoulder immobilized in a fixed sling for 2 weeks followed by 3 weeks in a non-fixed sling. The PR group starts loading (assisted active range of motion (AAROM) and active range of motion (AROM)) from week 2, while this is introduced in the UC group from week 6 . The PR group is attending individual physiotherapist-supervised exercise therapy three times weekly, supplemented with daily home exercises (week 2, 3, 4 and 5), and the UC group is attending individual physiotherapist-supervised exercise therapy once a week supplemented with daily home exercises (week 2, 3, 4 and 5). From weeks 6 to 12 all patients receive physiotherapist-supervised exercise therapy twice a week (individually or in small groups) next to the self-administered exercise once a week (Table 1). Descriptions of on-site and home interventions are found in the additional files (Additional files 1 and 2).

The exercise therapy program is tailored to each patient's capabilities at any given session. The therapists adjust exercise intensity as determined by the patient's ability to complete 3 sets of 10 repetitions for a given exercise without exacerbating pain. The overriding rule for all exercises is that pain above 5 on a numeric pain rating scale (NPRS) from 0 to 10 should not be provoked during exercises. The exercise therapy continuously determines and applies the load for 20 repetitions maximum (RM), with progression from $1 / 2 \mathrm{~kg}$ to $3-4 \mathrm{~kg}$ during the 12 weeks. Each exercise is guided with focus on correct performance and movement quality (direction, speed, posture and coordination) with sufficient rest between sets to allow for recovery.

It is recommended to increase the load by $2-10 \%$ when the patient can perform the current workload properly and with 1-2 repetitions more than the required number of 10 repetitions (Additional file 2). For both groups, scapular exercises are also performed with 20 RM, while stretching and mobility exercises are performed five times, for $20 \mathrm{~s}$ each. Both groups are instructed to complete a diary during the supervised sessions, including self-reported pain and use of pain medication as registered before and after each session.

\section{Treatment adherence}

Intervention adherence and attendance (on site and home-based) within the 12 weeks is recorded in exercise logbooks for both groups. In the exercise logbook, the patients are asked to report completed home-based exercise sessions and reasons for non-completed sessions (pain or other reasons). Supervision of the subsequent home exercises at the commencement of every session is performed to facilitate program adherence. Reinforcement techniques are used with the physiotherapist giving positive feedback and commending patients for their efforts.

As part of the post-operative standard medical treatment package all patients are offered paracetamol and non-steroidal anti-inflammatory drugs (NSAIDs) as basic pain treatment supplemented with morphine if necessary, and medication is prescribed before inclusion and randomization. Concomitant private physiotherapy sessions, acupuncture or other healthcare initiatives are prohibited during the trial. The available exercise equipment during the supervised rehabilitation includes Steens Physical Double Handle Speed Pulley (weight interval 0.5-2 kg) (Proterapi), Thera-Band System of progressive exercise, carpet tile, jump rope and broomstick. Also, sloping board (for supine elevation exercise in the scapular plane), ProFitter and various vinyl-coated hand weights produced by Trendy Sport will be available.

\section{Outcome measures/variables}

The primary (Western Ontario Rotator Cuff index, (WORC)) and secondary outcomes (Disability Arm Shoulder Hand (DASH), pain, ROM) are used at baseline (presurgery), 6 weeks, 12 weeks and 12 months after baseline 
Table 1 Overview of the postoperative intervention in main trial

\begin{tabular}{|c|c|c|c|}
\hline Week & Progressive rehabilitation (PR) & Week & Usual care (UC) \\
\hline $1-5$ & Shoulder immobilized in standard sling. & $1-5$ & Shoulder immobilized in standard sling. \\
\hline $2-5$ & $\begin{array}{l}\text { Physiotherapist guided PROM exercises } \\
\text { PROM Restrictions: } \\
\text { ABD + FLEX: None } \\
\text { IR }<90 \text { degrees in neutral } \\
\text { ER }<45 \text { degrees in neutral }\end{array}$ & $2-5$ & $\begin{array}{l}\text { Physiotherapist guided PROM exercises } \\
\text { PROM Restrictions: } \\
\text { ABD + FLEX: None } \\
\text { IR }<90 \text { degrees in neutral } \\
\text { ER }<45 \text { degrees in neutral }\end{array}$ \\
\hline 2 & $\begin{array}{l}\text { Close-chain AAROM and AROM exercises } \\
\text { AAROM and AROM Restrictions: } \\
A B D+F L E X<90 \text { degrees } \\
I R<90 \text { degrees in neutral } \\
\text { ER }=0 \text { degrees in neutral }\end{array}$ & & \\
\hline 3- 5 & $\begin{array}{l}\text { Close-chain AAROM and AROM exercises } \\
\text { AAROM and AROM Restrictions: } \\
A B D+F L E X<90 \text { degrees } \\
I R<90 \text { degrees in neutral } \\
\text { ER }<45 \text { degrees in neutral }\end{array}$ & & \\
\hline $6-12$ & $\begin{array}{l}\text { Therapist-supervised AROM (FLEX, ABD, EXT, ER and IR) } \\
\text { with gradually (individually) increased loading and } \\
\text { progression from close-chain to open-chain exercises. }\end{array}$ & $6-12$ & $\begin{array}{l}\text { Therapist-supervised AROM (FLEX, ABD, EXT, ER and IR) } \\
\text { with gradually (individually) increased loading and } \\
\text { progression from close-chain to open-chain exercises. }\end{array}$ \\
\hline $12-20$ & Continuation of rehabilitation in the community & $12-20$ & Continuation of rehabilitation in the community \\
\hline
\end{tabular}

$P R$ progressive rehabilitation, UC usual care, $R O M$ range of motion, $P R O M$ passive range of motion, $A B D$ abduction, FLEX flexion, IR Internal rotation, ER external rotation, $A A R O M$ assisted active range of motion, $A R O M$ active range of motion, EXT extension

(post-surgery) in addition to measurements of strength at baseline, 12 weeks and 12 months after baseline, and US measurements only 12 months after baseline (Fig. 2).

The primary outcome is the 12-week change in the WORC [47]. The WORC is a self-administered questionnaire developed (by interviewing patients) to measure health related quality of life (HRQL) in patients with rotator cuff disorders and it consists of 21 items in 5 domains: physical symptoms (6 items), sports and recreation (4 items), work (4 items), lifestyle (4 items) and emotions (3 items). Each question is scored on a $100 \mathrm{~mm}$ visual analogue scale (VAS) and summed to a total score for each domain, with a higher score indicating reduced quality of life. By inverting the raw score and then converting it to a percentage score $((2100-1875) / 2100 \times 100$ $=10.7 \%)$ ) each domain ranges from 0 (worst possible) to 100 (best possible) $[47,48]$. The WORC has excellent reliability with intra class correlation (ICC) between 0.84 and $0.99[49,50]$ and a minimal clinical important difference (MCID) of approximately $12 \%$ [47, 49, 51]. A recent translated and cross-culturally adapted Danish version was found valid in patients with rotator cuff tear (high correlation with the DASH (0.71), and test re-test reliable (ICC of 0.80 95\% CI 0.69-0.87)) [52].

Secondary patient-reported outcomes (PRO) include assessment of pain, functional activity level and HRQOL, using the DASH [53]. The DASH questionnaire is a self-administered questionnaire and is region-specific to upper extremity disorders and consists of 30 items divided into 6 items on symptoms and 24 on function. The questionnaire score ranges from 0 to 100 where 0 equals no disability and 100 equals the most severe disability [53]. The DASH is generated by asking questions of patients with upper extremity problems/disability and is valid, reliable and responsivene for this patient group $[53,54]$. The Danish validated version was to found to be a reliable [55] and responsive outcome measure in a variety of Danish-speaking patients with orthopedic upper extremity problems [56].

The Patient Global Rating Scale (GRS) is used to obtain a global/general impression of recovery from baseline to 6, 12 and 52 weeks postoperatively with the question: "Compared to when this treatment first started (before surgery), how would you describe your shoulder this last week?" This is assessed on a 15-point scale where -7 represents vastly worse, 0 represents unchanged, and +7 represents much better [57-59].

Patients are also asked about perceived pain using a NPRS [60, 61] with three questions: "On a scale from $0-10$, where 0 equals no pain and 10 the worst imaginable pain, how much pain do you feel in your shoulder during resting? / How much pain do you feel in your shoulder during activity? / What is the maximal shoulder pain you have experienced within the past 24 hours?"

Further, patients are asked to report use of analgesics (type (paracetamol or opioids)/amount). Secondary clinical outcomes consist of active and passive shoulder elevation ROM in the scapular plane (standing); external and internal shoulder rotation (in $90^{\circ}$ abduction when supine) $[62,63]$, as measured by digital inclinometer (Baseline Evaluation Instruments, model 12-1057 from Procare). Maximum voluntary isometric contraction (MVC) of 


\begin{tabular}{|c|c|c|c|c|c|c|c|}
\hline \multicolumn{6}{|c|}{ Visits besides pre screening and rehabilitation } & \multirow[t]{2}{*}{$\begin{array}{l}\text { Responsible } \\
\text { Personnel }\end{array}$} & \multirow{2}{*}{$\begin{array}{l}\text { Blinded to } \\
\text { group } \\
\text { allocation }\end{array}$} \\
\hline Visit & Baseline & Surgery & Week 6 & Week 12 & Week 52 & & \\
\hline Day \pm range & $-7 \pm 7$ & 1 & $40 \pm 3$ & $84 \pm 5$ & $364 \pm 14$ & & \\
\hline Assess eligibility & $\bullet$ & & & & & BHK & $\mathrm{N} / \mathrm{A}$ \\
\hline Informed consent & $\bullet$ & & & & & $\mathrm{BHK} / \mathrm{OA}$ & $\mathrm{N} / \mathrm{A}$ \\
\hline Randomization & & $\bullet$ & & & & SW & No \\
\hline WORC & $\bullet$ & & $\bullet$ & $\bullet$ & $\bullet$ & BHK & Yes \\
\hline DASH & $\bullet$ & & $\bullet$ & $\bullet$ & $\bullet$ & $\mathrm{BHK}$ & Yes \\
\hline GRS & & & $\bullet$ & $\bullet$ & $\bullet$ & BHK & Yes \\
\hline NPRS & $\bullet$ & & $\bullet$ & $\bullet$ & $\bullet$ & $\mathrm{BHK}$ & Yes \\
\hline ROM & $\bullet$ & & $\bullet$ & $\bullet$ & $\bullet$ & $\mathrm{BHK} / \mathrm{OA}$ & Yes \\
\hline Strength & $\bullet$ & & & $\bullet$ & $\bullet$ & $\mathrm{BHK} / \mathrm{OA}$ & Yes \\
\hline US & & & $\bullet$ & & & MD & Yes \\
\hline US (bilateral) & & & & & $\bullet$ & BHK & Yes \\
\hline Return to work & & & $\bullet$ & & $\bullet$ & $\mathrm{BHK}$ & Yes \\
\hline \multicolumn{8}{|c|}{$\begin{array}{l}\text { Fig. } 2 \text { Time schedule of enrollment, assessments and responsible trial personnel (Standard Protocol Items: Recommendation for Interventional } \\
\text { Trials (SPIRIT)). BHK, primary investigator; SW, co-author; N/A, not applicable; OA: outcome assessors; MD, medical doctor/ radiologist; WORC, } \\
\text { Western Ontario Rotator Cuff Index; DASH, Disabilities Arm, Shoulder and Hand questionnaire; MRI, magnetic resonance imaging; GRS, Global } \\
\text { Rating Scale; NPRS, numeric pain rating scale; ROM, range of motion; US, ultrasound }\end{array}$} \\
\hline
\end{tabular}

shoulder external and internal rotation (sitting) and elevation in the scapular plane $[62,64,65]$ is measured by dynamometer (IsoForceControl, model EVO2, 10-400 N, Medical Device Solutions AG). Furthermore, intraoperative information (including details of tendon repair) and patient-reported number of sick-leave days from work/time until return to work and leisure activities are registered.

Tendon healing characteristics of the repaired supraspinatus tendon (SST) and the subacromial space (SAS) are investigated at 12 months follow-up using US greyscale. The SAS and the SST thickness are also potentially important factors in understanding the pathogenesis of rotator cuff pathology $[66,67]$, tendinopathy $[68]$ and ruptures [69] a year after repair [70]. Furthermore, power Doppler (PD) US is used for measuring vascularization as a sign of pathology and healing [70, 71]. The US examination is performed by the principal investigator (BHK) using a Hitachi Ascendus scanner and Hitachi Medical Systems Linear standard probe type L75 $18-5 \mathrm{MHz}$ 50-mm transducer (Hitachi Aloka Medical) and the musculoskeletal program. The depth preset is $2.50 \mathrm{~cm}$ and focus is adjusted, so it is placed right under the tendon.

Thus, the US investigation at 12 months involves quantitative measures of the repaired and contralateral SST thickness and neovascularity (NV), in addition to the SAS measured as the acromiohumeral distance (AHD). Ultrasound images are captured with the patients seated in an upright position, feet flat on the floor, neutral trunk posture, and head facing forward. For the SST thickness measure the patient has the hand on the affected side on the ipsilateral posterior hip with the humerus in extension and the tendon is measured in a longitudinal [72] and a transverse view [67]. AHD is measured in the neutral position (arm resting at the side) and respectively with $45^{\circ}$ and $60^{\circ}$ of active shoulder elevation in the scapular plane [72, 73]. SST neovascularity assessed by power Doppler is measured with the shoulder internally rotated with the dorsal side of the hand placed on the sacrum, and the elbow flexed and directed laterally [74]. 
Registered demographic data include age, gender, tendon(s) involved, supplementary surgery performed, hand dominance, history of trauma, occupation/employment and preoperative sports/recreational activity level. Demographic data and baseline measures will be used in the analysis of predictors of health outcomes. The investigator or site personnel monitor each patient for adverse events (AEs) throughout the study. Tendon re-tear is assessed by US at 6 weeks postoperatively and any re-tear is recorded. The investigator assesses and records any $\mathrm{AE}$ (in response to a query, observed by site personnel or reported spontaneously by the patient) in detail, including the date of onset, description, severity, duration and outcome, relationship of the $\mathrm{AE}$ to study intervention and any action(s) taken. All AEs will be followed closely to make sure that the patient is safely handled including follow up on clinical issues, until a stable situation is reached.

\section{Data collection}

The primary investigator performs all enrollment after screening by the orthopedic surgeons. The primary investigator and two outcome assessors perform all baseline and follow-up assessments. Before starting the data collection, the assessors and the primary investigator decide on a consensus standard for collection and recording of all outcome variables. We use the Procordo Research Platform, which is an electronic online data trial management system (DTMS) (www.procordo.com) to collect and store the data. In the system the patients answer the questionnaires in a web-based survey form, and the outcome assessor manually registers data from the objective assessments. The DTMS includes automatic range checks of the entered data values, and the user will be alerted if the entered data fall outside a pre-specified range.

\section{Sample size and power considerations}

The sample size is calculated to test the superiority of the PR protocol over the UC protocol in the assessment of change in the WORC physical symptoms subscale at week 12 (primary outcome) [75]. With 41 patients per group, the study will have $80 \%$ power assuming the expected group difference in the mean changes from baseline is 12 points, corresponding to the suggested minimum clinical relevant difference [47], the common standard deviation is $20(0-100$ scale) [75] and a significance level of $5 \%$. With an expected dropout around $20 \%$ during the study we will randomize and allocate 100 patients (50 to each group); analyzing the intention to treat (ITT) population, which will give power of $85 \%$ to detect the aforementioned difference. A provisional deadline for patient recruitment is set to June 2018, but in case the target number of 100 patients has not been met the recruitment period may be extended to reach the number required (2 times 41 patients) to obtain power of at least $0.8(80 \%)$.

\section{Allocation, randomization and sequence generation}

After baseline tests and surgery the patients will be randomized equally (1:1) to receive either the PR protocol or the UC protocol. The computer-generated randomization to one of two treatment arms is performed based upon permuted random blocks of variable size (4-8 in each block) using the Procordo Research Platform. To counter potential imbalance in the randomization, stratification by trial site and age ( $+/-57$ years) will be employed together with blocking. The randomization is performed by a person $(\mathrm{SW})$ with no clinical involvement in the trial, and the person notifies the patient and clinical study staff of the treatment allocation. The allocation will be concealed in a password-protected Research Platform only accessible by the senior researcher and the independent data manager (Procordo).

\section{Blinding}

Orthopedic surgeons will perform initial screening. As this is an "open-label" trial the health professionals delivering the interventions and the patients will not be blinded to treatment allocation. The principal investigator and outcome assessors will be blinded to treatment allocation during all pre-examinations and post-examinations, and patients are requested not to disclose their allocation when outcomes are assessed at weeks 6 and 12 and 12 months postoperatively. To test the efficacy of blinding, the outcome assessors are asked what treatment strategy they think a patient has received after assessments at week 12 postoperatively. The principal investigator and outcome assessors can be un-blinded if deemed necessary, e.g. in the case of (serious) adverse events that require these otherwise blinded persons to be involved in the solution of the event.

\section{Statistical analysis plan}

The primary efficacy analysis performed is assessment of the between-group difference in change in the WORC score after 12 weeks in the ITT population. The ITT population is defined as all randomized patients irrespective of compliance or withdrawals. Missing data will be replaced using a multiple imputation technique with age, gender, center, group allocation (masked) and baseline values as predictors. For sensitivity purposes missing data will be imputed using baseline observation carried forward (BOCF). The modified ITT population is defined as the ITT population that has a valid baseline observation of the variable of interest. The as-observed (AO) population is defined as patients who have the outcome of interest assessed at a given time point of interest (i.e. no imputation of missing data will be done).

The per-protocol (PP) population is defined as patients in the AO population that adhere to this protocol, defined by the criteria of the two groups: the PR group (Table 1), 
included in the AO population and have attended at least $75 \%$ of the scheduled rehabilitation appointments, whether at clinical visits or home-based as tailored to the individual (documented by the exercise logbook), and do not stop the intervention during the 12-week main trial period and do not engage in concomitant exercise therapy (e.g. private physiotherapy sessions, acupuncture); and the UC group (Table 1), included in the AO population, and do not stop the intervention during the 12-week main trial period and do not engage in concomitant exercise therapy. Each patient registers the home shoulder exercises in an exercise logbook, and successful completion of the intervention is defined as performance of $75 \%$ of the planned exercises.

The primary analysis at week 12 will be by analysis of covariance (ANCOVA) on change from baseline in the WORC domains as dependent variables, with group allocation (two levels) and time (week 6 and 12), gender and site as fixed factors, whereas age and baseline values will be analyzed as covariates. Secondary outcome measures will be analyzed by multiple logistic regression for binary outcome measures (re-tear, tendon healing, recurrence of symptoms (e.g. pain and function), complications) and by ANCOVA for the continuous outcome measures (pain, patient-reported outcomes, surgery information, strength, ROM) as dependent variables (individually), with group allocation and time as fixed factors, and site and baseline values as covariates. Predictors of health outcomes will be analyzed by ANCOVA or multiple regression as independent variables, with change in the WORC as the dependant variable. All statistical tests will be two-sided and statistical significance will be denoted by a computed $p$ value equal to or less than 0.05 . All data analysis will be carried out according to a pre-established analysis plan. The statistical analysis plan (SAP) will be carried through blinded according to group allocation, and results will be interpreted in an author consensus statement prior to disclosing/revealing group allocation on the basis of a blinded review of the data from the primary endpoint (changes from treatment A compared to changes from treatment B), assuming that treatment $\mathrm{A}$ is the active treatment (PR), and the other assuming that treatment $\mathrm{B}$ is the active treatment (UC). Analysis of the primary outcome will be calculated blinded, and two interpretations will be performed based on whether PR or UC is superior. Not until signed consent from all of the authors of this trial (identical to the authors of this SAP) has been obtained, agreeing on one interpretation of the results only, will the randomization code be broken. This is to reduce bias in the interpretation of the current findings. On agreement, all members of the author group will approve and sign the interpretations before any publication procedures are initiated [76].
The protocol conforms to the recommendations of the Enhancing the Quality and Transparency Of health Research (EQUATOR) network [77] using the Standard Protocol Items: Recommendations for Interventional Trials (SPIRIT) checklist and the Consolidating Standards of Reporting Trials (CONSORT) statement [78] (Additional file 3).

\section{Interim analysis and early stopping rules}

An early stopping rule will be applied when there is complete rotator cuff re-tears corresponding to at least $25 \%$ of the calculated number in the PR group as verified by ultrasonography 6 weeks postoperatively, provided that the re-tear rate is more than twice as high compared to that in the control UC group [79]. The trial steering committee (the authors of this manuscript) decide if the early stopping rule should be effectuated.

\section{Safety monitoring}

No data monitoring committee has been established since adverse events are expected to be minimal and the intervention is not considered to be high risk. The physiotherapists at each center will report any adverse event to the primary investigator, who reports these to the ethics committee. The person responsible for randomization will monitor if the number of adverse events has reached the threshold for the early stopping rule. Further, if a patient experiences extreme worsening of symptoms during exercise therapy and these symptoms have not subsided before the next exercise session, the physiotherapist will report it to the primary investigator, who may refer the patient to an orthopedic specialist for an evaluation. The number and seriousness of AEs will be reported.

\section{Ethics}

Prior to screening, all potential trial patients are informed orally and in writing about the purpose of this trial, its process and potential risks and the costs and benefits of participation. All patients are informed of their rights to withdraw from the study at any time without this impacting on any future investigations and/or treatments at any site or by any member inside or outside the study staff. Informed consent will be obtained from all patients. All data will be handled in confidence according to the Danish Data Protection Act. The study was approved by Health Research Study Board for the Capital Region Denmark (H-16033995) on the 18 October 2016 and by the Danish Data Protection Agency (2012-58-0004) on the 15 February 2017 and the study was registered at www.clinicaltrials.gov (NCT02969135) on the 15 November 2016. The study will be conducted in accordance with Danish law, the local research ethics committee requirements and the principles of the Declaration of Helsinki [80]. Positive as well as negative and inconclusive results will be published 
in scientific peer-reviewed journals, with authorship following the International Committee of Medical Journal Editors (ICMJE) guidelines for publication. The results will be presented orally nationally and internationally. Upon full publication of the dataset by the investigators, we intend to share the data for future research purposes.

\section{Discussion}

The effect of rehabilitation after rotator cuff surgery has been discussed during the past 10 years and 13 systematic reviews including meta-analyses or systematic review of overlapping meta-analyses have been published since 2014 including the same few (8-12) primary studies [21-26, 81-87]. These have focused on early ROM exercises and immobilization periods. Nevertheless, there are only a few clinical studies on postoperative treatment of individuals with rotator cuff repair and the existing studies are of moderate to low quality. This is primarily due to small sample sizes, inadequate blinding of patients and/or investigators and incomplete intervention descriptions [15-20], making it difficult to transform the results into clinical practice. Furthermore, previous studies use a wide range of clinical and patient-reported outcomes, generally with poor responsiveness and per-protocol analyses. However, collectively there is evidence that early intervention is advantageous in the rehabilitation because early ROM exercises accelerate healing, reduce stiffness, do not increase risk of re-tear, and because immobilization does not increase tendon healing or clinical outcome [21-24].

The CUT-N-MOVE trial will add new knowledge to the field with regards to the effect of an early and targeted progressive treatment program for patients who had surgery due to a rotator cuff tear. To our knowledge this study is the first evaluating the combined effect of early (time of initiation) and progressive (intensity, also including active muscle contraction) postoperative exercises on parameters such as physical function, pain, quality of life and return to work.

Inclusion criteria in this study are broad with respect to age and concurrent diseases in an attempt to reflect clinical practice. However, inclusion criteria are narrow regarding the specific condition, and thus we require the tears to be based on relevant extrinsic trauma and at the least a complete supraspinatus tear must be verified during surgery/arthroscopy.

The present exercise program focuses in several ways (passive, assisted active and active ROM exercises and closed and open chain exercises) on each individual patient's capability on the day. Further, this program differs, to our knowledge, from previously outlined exercise programs in having progressive but gently assisted active exercise starting as soon as the second week postoperatively. Importantly, in addition to functional outcomes (considered the most relevant for the patients) we also follow the pathological and mechanical tendon-healing process compared with the contralateral side.

The aim of the CUT-N-MOVE trial is to investigate whether there is a significant advantage in using progressive early passive and active exercise therapy compared with a traditional, limited early passive exercise therapy in patients recovering from surgical treatment of rotator cuff tears. If this is the case, the consequence is that clinicians will have a detailed description of a step-wise progressive rehabilitation program available when treating patients who have had surgery due to a rotator cuff tear.

\section{Trial status}

At the time of submission of this study protocol the trial is ongoing and still recruiting patients. The recruitment of patients began in February 2017 and will continue until the complete sample size is achieved, which is expected in June 2018 .

\section{Additional files}

Additional file 1: Exercise therapy program for the CUT-N-MOVE trial. (PDF 53848 kb)

Additional file 2: Exercise progression for the CUT-N-MOVE trial. (PDF $234 \mathrm{~kb}$ )

Additional file 3: The SPIRIT checklist for the CUT-N-MOVE trial. (PDF $99 \mathrm{~kb}$ )

\section{Abbreviations}

AAROM: Assisted active range of motion; ABD: Abduction; AE: Adverse event: AHD: Acromiohumeral distance; ANCOVA: Analysis of covariance; AO: As observed; AROM: Active range of motion; BOCF: Baseline observation carried forward; CONSORT: Consolidating Standards Of Reporting Trials;

DASH: Disabilities Arm, Shoulder and Hand questionnaire; EQUATOR: Enhancing the quality and transparency of health research; ER: External rotation; EXT: Extension; FLEX: Flexion; GRS: Global Rating Scale; HRQOL: Health related quality of life; ICC: Intra class correlation; IR: Internal rotation; ITT: Intention to treat; MCID: Minimal clinical important difference; MVC: Maximal voluntary contraction; NPRS: Numeric Pain Rating Scale; NV: Neovascularity; PD: Power Doppler; PP: Per protocol; PR: Progressive rehabilitation; PRO: Patient-reported outcomes; PROM: Passive range of motion; RC: Rotator cuff; ROM: Range of motion; SAP: Statistical analysis plan; SAS: Subacromial space; SPIRIT: Standard Protocol Items: Recommendation For Interventional Trials; SST: Supraspinatus tendon; UC: Usual care;

US: Ultrasound; VAS: Visual analogue scale; WORC: Western Ontario Rotator Cuff Index

\section{Acknowledgements}

We thank all participating orthopedic specialists in performing the initial screening of patients and physiotherapists in pre-testing and post-testing patients. Also thanks to the physiotherapists who handle the rehabilitation by instructing patients in the exercises, and the participating departments at Bispebjerg-Frederiksberg Hospital and Herlev-Gentofte Hospital,

Copenhagen who contribute personnel.

\section{Funding}

The trial is mainly funded by grants from The Danish Physiotherapy Association, The Faculty of Health Sciences at University of Southern Denmark and The

Danish Rheumatism Association. Internal financial, administrative and personnel support is received throughout the entire trial from the Department of Physical and Occupational Therapy, Bispebjerg-Frederiksberg Hospital. 


\section{Authors' contributions}

BHK designed and initiated the trial in collaboration with BJK and PM. BHK applied for funding, established collaborations, is responsible for logistics, patient recruitment, data collection and leading the coordination of the trial and has prepared the first draft of this manuscript. BJK, PM, SW, MK and $\mathrm{MH}$ assisted in trial design, the statistical analysis plan and writing of this manuscript. All authors were involved in drafting the manuscript and critically revising it for important intellectual content and, finally, read and approved the final manuscript.

\section{Ethics approval and consent to participate}

The Health Research Study Board for the Capital Region Denmark approved the study on the 18 October 2016 (H-16033995). The study will be conducted in accordance with the local research ethics committee requirements and the principles of the Declaration of Helsinki, thus informed consent will be obtained from all study participants.

\section{Consent for publication}

Informed consent for publication of identifiable human images provided in the additional files has been obtained.

\section{Competing interests}

The authors declare that they have no competing interests.

\section{Publisher's Note}

Springer Nature remains neutral with regard to jurisdictional claims in published maps and institutional affiliations.

\section{Author details}

${ }^{1}$ Department of Physical and Occupational Therapy, Bispebjerg-Frederiksberg Hospital, Bispebjerg Bakke 23, DK-2400 Copenhagen, Denmark. ²Department of Sports Science and Clinical Biomechanics, University of Southern Denmark, Campusvej 55, DK-5230 Odense, Denmark. ${ }^{3}$ Institute of Sports Medicine, Department of Orthopaedic Surgery M, Copenhagen Bispebjerg-Frederiksberg Hospital, and Center for Healthy Aging, Faculty of Health and Medical Sciences, University of Copenhagen, Copenhagen, Denmark. ${ }^{4}$ Section for Sports Traumatology M51, Department of Orthopaedic Surgery, Bispebjerg-Frederiksberg Hospital, Bispebjerg Bakke 23, DK-2400 Copenhagen, Denmark. ${ }^{5}$ The Parker Institute, Bispebjerg-Frederiksberg Hospital, Ndr. Fasanvej 57, DK-2000 Frederiksberg, Copenhagen, Denmark.

\section{Received: 12 February 2018 Accepted: 4 August 2018}

\section{Published online: 03 September 2018}

\section{References}

1. Lewis JS. Rotator cuff tendinopathy/subacromial impingement syndrome: is it time for a new method of assessment? Br J Sports Med. 2009;43:259-64.

2. Luime JJ, Koes BW, Hendriksen IJ, Burdorf A, Verhagen AP, Miedema HS, Verhaar JA. Prevalence and incidence of shoulder pain in the general population; a systematic review. Scand J Rheumatol. 2004;33:73-81.

3. van der Windt DA, Koes BW, de Jong BA, Bouter LM. Shoulder disorders in general practice: incidence, patient characteristics, and management. Ann Rheum Dis. 1995;54:959-64.

4. Huisstede BM, Wijnhoven HA, Bierma-Zeinstra SM, Koes BW, Verhaar JA, Picavet S. Prevalence and characteristics of complaints of the arm, neck, and/or shoulder (CANS) in the open population. Clin J Pain. 2008;24:253-9.

5. Kuijpers T, van der Windt DA, van der Heijden GJ, Twisk JW, Vergouwe Y, Bouter LM. A prediction rule for shoulder pain related sick leave: a prospective cohort study. BMC Musculoskelet Disord. 2006;7:97.

6. Lotters F, Meerding WJ, Burdorf A. Reduced productivity after sickness absence due to musculoskeletal disorders and its relation to health outcomes. Scand J Work Environ Health. 2005;31:367-74.

7. Kukkonen J, Joukainen A, Itala A, Aarimaa V. Operatively treated traumatic versus non-traumatic rotator cuff ruptures: a registry study. Ups J Med Sci. 2013;118:29-34.

8. Fehringer EV, Sun J, VanOeveren LS, Keller BK, Matsen FA 3rd. Fullthickness rotator cuff tear prevalence and correlation with function and co-morbidities in patients sixty-five years and older. J Shoulder Elb Surg. 2008;17:881-5.
9. Hawkins RJ, Morin WD, Bonutti PM. Surgical treatment of fullthickness rotator cuff tears in patients 40 years of age or younger. J Shoulder Elb Surg. 1999:8:259-65.

10. Mazzocca AD, Rincon LM, O'Connor RW, Obopilwe E, Andersen M, Geaney L, Arciero RA. Intra-articular partial-thickness rotator cuff tears: analysis of injured and repaired strain behavior. Am J Sports Med. 2008:36:110-6.

11. Gerber C, Fuchs B, Hodler J. The results of repair of massive tears of the rotator cuff. J Bone Joint Surg Am. 2000;82:505-15.

12. Ensor KL, Kwon YW, Dibeneditto MR, Zuckerman JD, Rokito AS. The rising incidence of rotator cuff repairs. J Shoulder Elb Surg. 2013;22:1628-32

13. Thorpe A, Hurworth M, O'Sullivan P, Mitchell T, Smith A. Rising trends in surgery for rotator cuff disease in Western Australia. ANZ J Surg. 2016;86:801-4.

14. National Klinisk Retningslinje for diagnostik og behandling af patienter med udvalgte skulderlidelser [https://www.sst.dk/da/udgivelser/2013/ /media/ ECDF89CE7B904A34A5EC8576B507289D.ashx]. Accessed 17 Aug 2018.

15. Duzgun I, Baltaci G, Atay OA. Comparison of slow and accelerated rehabilitation protocol after arthroscopic rotator cuff repair: pain and functional activity. Acta Orthop Traumatol Turc. 2011;45:23-33.

16. Arndt J, Clavert P, Mielcarek P, Bouchaib J, Meyer N, Kempf JF. Immediate passive motion versus immobilization after endoscopic supraspinatus tendon repair: a prospective randomized study. Orthop Traumatol Surg Res. 2012;98:S131-8.

17. Cuff DJ, Pupello DR. Prospective randomized study of arthroscopic rotator cuff repair using an early versus delayed postoperative physical therapy protocol. J Shoulder Elb Surg. 2012;21:1450-5.

18. Lee $B G$, Cho NS, Rhee YG. Effect of two rehabilitation protocols on range of motion and healing rates after arthroscopic rotator cuff repair: aggressive versus limited early passive exercises. Arthroscopy. 2012;28:34-42.

19. Keener JD, Galatz LM, Stobbs-Cucchi G, Patton R, Yamaguchi K. Rehabilitation following arthroscopic rotator cuff repair: a prospective randomized trial of immobilization compared with early motion. J Bone Joint Surg Am. 2014;96:11-9.

20. Kim YS, Chung SW, Kim JY, Ok JH, Park I, Oh JH. Is early passive motion exercise necessary after arthroscopic rotator cuff repair? Am J Sports Med. 2012;40:815-21.

21. Chan K, MacDermid JC, Hoppe DJ, Ayeni OR, Bhandari M, Foote CJ, Athwal GS. Delayed versus early motion after arthroscopic rotator cuff repair: a meta-analysis. J Shoulder Elb Surg. 2014;23:1631-9.

22. Chang KV, Hung CY, Han DS, Chen WS, Wang TG, Chien KL. Early versus delayed passive range of motion exercise for arthroscopic rotator cuff repair: a meta-analysis of randomized controlled trials. Am J Sports Med. 2014:43:1265-73.

23. Riboh JC, Garrigues GE. Early passive motion versus immobilization after arthroscopic rotator cuff repair. Arthroscopy. 2014;30:997-1005.

24. Shen C, Tang ZH, Hu JZ, Zou GY, Xiao RC, Yan DX. Does immobilization after arthroscopic rotator cuff repair increase tendon healing? A systematic review and meta-analysis. Arch Orthop Trauma Surg. 2014;134:1279-85.

25. Littlewood C, Bateman M, Clark D, Selfe J, Watkinson D, Walton M, Funk L. Rehabilitation following rotator cuff repair: a systematic review. Shoulder Elbow. 2015;7:115-24.

26. Thomson S, Jukes C, Lewis J. Rehabilitation following surgical repair of the rotator cuff: a systematic review. Physiotherapy. 2016;102:20-8.

27. Strauss EJ, Salata MJ, Kercher J, Barker JU, McGill K, Bach BR Jr, Romeo AA Verma NN. Multimedia article. The arthroscopic management of partialthickness rotator cuff tears: a systematic review of the literature. Arthroscopy. 2011;27:568-80.

28. DeHaan AM, Axelrad TW, Kaye E, Silvestri L, Puskas B, Foster TE. Does double-row rotator cuff repair improve functional outcome of patients compared with single-row technique? A systematic review. Am J Sports Med. 2012:40:1176-85.

29. Sheibani-Rad S, Giveans MR, Arnoczky SP, Bedi A. Arthroscopic single-row versus double-row rotator cuff repair: a meta-analysis of the randomized clinical trials. Arthroscopy. 2013;29:343-8.

30. Burkhart SS. Arthroscopic treatment of massive rotator cuff tears. Clin Orthop Relat Res. 2001:107-18.

31. Coghlan JA, Buchbinder R, Green S, Johnston RV, Bell SN. Surgery for rotator cuff disease. Cochrane Database Syst Rev. 2008:CD005619.

32. Davidson J, Burkhart SS. The geometric classification of rotator cuff tears: a system linking tear pattern to treatment and prognosis. Arthroscopy. 2010;26:417-24.

33. Halder AM, O'Driscoll SW, Heers G, Mura N, Zobitz ME, An KN Kreusch-Brinker R. Biomechanical comparison of effects of 
supraspinatus tendon detachments, tendon defects, and muscle retractions. J Bone Joint Surg Am. 2002;84-A:780-5.

34. Hatakeyama $Y$, Itoi E, Pradhan RL, Urayama M, Sato K. Effect of arm elevation and rotation on the strain in the repaired rotator cuff tendon. A cadaveric study. Am J Sports Med. 2001;29:788-94.

35. Kannus $P$, Jozsa L, Kvist M, Lehto M, Jarvinen M. The effect of immobilization on myotendinous junction: an ultrastructural, histochemical and immunohistochemical study. Acta Physiol Scand. 1992;144:387-94.

36. Kannus $P$, Jozsa $L$, Natri A, Jarvinen $M$. Effects of training, immobilization and remobilization on tendons. Scand J Med Sci Sports. 1997;7:67-71.

37. Michna H. Morphometric analysis of loading-induced changes in collagenfibril populations in young tendons. Cell Tissue Res. 1984;236:465-70.

38. Lastayo PC, Wright T, Jaffe R, Hartzel J. Continuous passive motion after repair of the rotator cuff. A prospective outcome study. J Bone Joint Surg Am. 1998;80:1002-11.

39. Thomopoulos S, Williams GR, Soslowsky LJ. Tendon to bone healing: differences in biomechanical, structural, and compositional properties due to a range of activity levels. J Biomech Eng. 2003;125:106-13.

40. Woo SL, Gomez MA, Woo YK, Akeson WH. Mechanical properties of tendons and ligaments. II. The relationships of immobilization and exercise on tissue remodeling. Biorheology. 1982;19:397-408.

41. Koo SS, Parsley BK, Burkhart SS, Schoolfield JD. Reduction of postoperative stiffness after arthroscopic rotator cuff repair: results of a customized physical therapy regimen based on risk factors for stiffness. Arthroscopy. 2011;27:155-60.

42. Parsons BO, Gruson Kl, Chen DD, Harrison AK, Gladstone J, Flatow EL. Does slower rehabilitation after arthroscopic rotator cuff repair lead to long-term stiffness? J Shoulder Elb Surg. 2010;19:1034-9.

43. Reilly P, Bull AM, Amis AA, Wallace AL, Richards A, Hill AM, Emery RJ. Passive tension and gap formation of rotator cuff repairs. J Shoulder Elb Surg. 2004;13:664-7.

44. Park MC, Jun BJ, Park CJ, Ahmad CS, ElAttrache NS, Lee TQ. The biomechanical effects of dynamic external rotation on rotator cuff repair compared to testing with the humerus fixed. Am J Sports Med. 2007;35:1931-9.

45. Debeyre J, Patie D, Elmelik E. Repair of ruptures of the rotator cuff of the shoulder. J Bone Joint Surg Br. 1965;47:36-42.

46. de Boer MD, Maganaris CN, Seynnes OR, Rennie MJ, Narici MV. Time course of muscular, neural and tendinous adaptations to 23 day unilateral lowerlimb suspension in young men. J Physiol. 2007:583:1079-91.

47. Kirkley A, Alvarez C, Griffin S. The development and evaluation of a diseasespecific quality-of-life questionnaire for disorders of the rotator cuff: the western Ontario rotator cuff index. Clin J Sport Med. 2003;13:84-92.

48. MacDermid JC, Drosdowech D, Faber K. Responsiveness of self-report scales in patients recovering from rotator cuff surgery. J Shoulder Elb Surg. 2006; 15:407-14.

49. Ekeberg OM, Bautz-Holter E, Tveita EK, Keller A, Juel NG, Brox Jl. Agreement, reliability and validity in 3 shoulder questionnaires in patients with rotator cuff disease. BMC Musculoskelet Disord. 2008:9:68.

50. Lopes AD, Ciconelli RM, Carrera EF, Griffin S, Faloppa F, Dos Reis FB. Validity and reliability of the western Ontario rotator cuff index (WORC) for use in Brazil. Clin J Sport Med. 2008;18:266-72.

51. Zhaeentan S, Legeby M, Ahlstrom S, Stark A, Salomonsson B. A validation of the Swedish version of the WORC index in the assessment of patients treated by surgery for subacromial disease including rotator cuff syndrome. BMC Musculoskelet Disord. 2016;17:165

52. Brix LD, Thillemann TM, Biørnholdt KT, Nikolajsen L. Cross-cultural translation, validation, reliability and responsiveness of the Danish version of Western Ontario Rotator Cuff Index. Abstract Proceeding. 2018. http://dssak. ortopaedi.dk/wp-content/uploads/2018/04/DSSAK-Annual-Meeting-2018final-program.pdf. Accessed 20 Apr 2018.

53. Hudak PL, Amadio PC, Bombardier C. Development of an upper extremity outcome measure: the DASH (disabilities of the arm, shoulder and hand) [corrected]. The upper extremity collaborative group (UECG). Am J Ind Med. 1996;29:602-8.

54. Beaton DE, Katz JN, Fossel AH, Wright JG, Tarasuk V, Bombardier C. Measuring the whole or the parts? Validity, reliability, and responsiveness of the disabilities of the arm, shoulder and hand outcome measure in different regions of the upper extremity. J Hand Ther. 2001;14:128-46.

55. Herup A, Merser S, Boeckstyns M. Validation of questionnaire for conditions of the upper extremity. Ugeskr Laeger. 2010;172:3333-6.
56. Lundquist CB, Dossing K, Christiansen DH. Responsiveness of a Danish version of the disabilities of the arm, shoulder and hand (DASH) questionnaire. Dan Med J. 2014;61:A4813.

57. de Vet HC, Terwee CB, Ostelo RW, Beckerman H, Knol DL, Bouter LM. Minimal changes in health status questionnaires: distinction between minimally detectable change and minimally important change. Health Qual Life Outcomes. 2006;4:54.

58. Bernard JA, Dattilo JR, Srikumaran U, Zikria BA, Jain A, LaPorte DM. Reliability and validity of 3 methods of assessing orthopedic resident skill in shoulder surgery. J Surg Educ. 2016;73:1020-5.

59. Revicki D, Hays RD, Cella D, Sloan J. Recommended methods for determining responsiveness and minimally important differences for patient-reported outcomes. J Clin Epidemiol. 2008:61:102-9.

60. Downie WW, Leatham PA, Rhind VM, Wright V, Branco JA, Anderson JA. Studies with pain rating scales. Ann Rheum Dis. 1978;37:378-81.

61. Mintken PE, Glynn P, Cleland JA. Psychometric properties of the shortened disabilities of the arm, shoulder, and hand questionnaire (QuickDASH) and numeric pain rating scale in patients with shoulder pain. J Shoulder Elb Surg. 2009;18:920-6.

62. Cools AM, De Wilde L, Van Tongel A, Ceyssens C, Ryckewaert R, Cambier DC. Measuring shoulder external and internal rotation strength and range of motion: comprehensive intra-rater and inter-rater reliability study of several testing protocols. J Shoulder Elb Surg. 2014;23:1454-61.

63. de Winter AF, Heemskerk MA, Terwee CB, Jans MP, Deville W, van Schaardenburg DJ, Scholten RJ, Bouter LM. Inter-observer reproducibility of measurements of range of motion in patients with shoulder pain using a digital inclinometer. BMC Musculoskelet Disord. 2004;5:18.

64. Cadogan A, Laslett M, Hing W, McNair P, Williams M. Reliability of a new hand-held dynamometer in measuring shoulder range of motion and strength. Man Ther. 2011;16:97-101.

65. Edouard P, Samozino P, Julia M, Gleizes Cervera S, Vanbiervliet W, Calmels P, Gremeaux $V$. Reliability of isokinetic assessment of shoulder-rotator strength: a systematic review of the effect of position. J Sport Rehabil. 2011;20:367-83.

66. Seitz AL, Michener LA. Ultrasonographic measures of subacromial space in patients with rotator cuff disease: a systematic review. J Clin Ultrasound. 2011;39:146-54.

67. Michener LA, Subasi Yesilyaprak SS, Seitz AL, Timmons MK, Walsworth MK. Supraspinatus tendon and subacromial space parameters measured on ultrasonographic imaging in subacromial impingement syndrome. Knee Surg Sports Traumatol Arthrosc. 2015;23:363-9.

68. Sunding K, Fahlstrom M, Werner S, Forssblad M, Willberg L. Evaluation of Achilles and patellar tendinopathy with greyscale ultrasound and colour Doppler: using a four-grade scale. Knee Surg Sports Traumatol Arthrosc. 2016;24:1988-96.

69. Teefey SA, Middleton WD, Bauer GS, Hildebolt CF, Yamaguchi K Sonographic differences in the appearance of acute and chronic fullthickness rotator cuff tears. J Ultrasound Med. 2000;19:377-8. quiz 383

70. Eliasson P, Couppe C, Lonsdale M, Svensson RB, Neergaard C, Kjaer M, Friberg L, Magnusson SP. Ruptured human Achilles tendon has elevated metabolic activity up to 1 year after repair. Eur J Nucl Med Mol Imaging. 2016;43:1868-77.

71. Poltawski L, Ali S, Jayaram V, Watson T. Reliability of sonographic assessment of tendinopathy in tennis elbow. Skelet Radiol. 2012:41:83-9.

72. Kjær BH, Ellegaard K, Wieland I, Warming S, Juul-Kristensen B. Intra-rater and inter-rater reliability of the standardized ultrasound protocol for assessing subacromial structures. Physiother Theory Pract. 2017;33:398-409.

73. Desmeules F, Minville L, Riederer B, Cote CH, Fremont P. Acromiohumeral distance variation measured by ultrasonography and its association with the outcome of rehabilitation for shoulder impingement syndrome. Clin J Sport Med. 2004;14:197-205

74. Ingwersen KG, Hjarbaek J, Eshoej H, Larsen CM, Vobbe J, JuulKristensen B. Ultrasound assessment for grading structural tendon changes in supraspinatus tendinopathy: an inter-rater reliability study. BMJ Open. 2016:6:e011746.

75. de Witte PB, Henseler JF, Nagels J, Vliet Vlieland TP, Nelissen RG. The western Ontario rotator cuff index in rotator cuff disease patients: a comprehensive reliability and responsiveness validation study. Am J Sports Med. 2012;40:1611-9.

76. Jarvinen $\mathrm{TL}$, Sihvonen $\mathrm{R}$, Bhandari M, Spraque S, Malmivaara A, Paavola $\mathrm{M}$, Schunemann $\mathrm{HJ}$, Guyatt $\mathrm{GH}$. Blinded interpretation of study results can feasibly and effectively diminish interpretation bias. J Clin Epidemiol. 2014;67:769-72. 
77. Christensen R, Bliddal H, Henriksen M. Enhancing the reporting and transparency of rheumatology research: a guide to reporting guidelines. Arthritis Res Ther. 2013;15:109.

78. Moher D, Hopewell S, Schulz KF, Montori V, Gotzsche PC, Devereaux PJ, Elbourne D, Egger M, Altman DG, Consolidated Standards of Reporting Trials G. CONSORT 2010 Explanation and elaboration: updated guidelines for reporting parallel group randomised trials. J Clin Epidemiol. 2010, 63:e1-37.

79. McElvany MD, McGoldrick E, Gee AO, Neradilek MB, Matsen FA 3rd. Rotator cuff repair: published evidence on factors associated with repair integrity and clinical outcome. Am J Sports Med. 2015:43:491-500.

80. Vollmann J, Winau R. Informed consent in human experimentation before the Nuremberg code. BMJ. 1996;313:1445-9.

81. Chen L, Peng K, Zhang D, Peng J, Xing F, Xiang Z. Rehabilitation protocol after arthroscopic rotator cuff repair: early versus delayed motion. Int J Clin Exp Med. 2015;8:8329-38.

82. Kluczynski MA, Nayyar S, Marzo JM, Bisson LJ. Early versus delayed passive range of motion after rotator cuff repair: a systematic review and metaanalysis. Am J Sports Med. 2015;43:2057-63.

83. Yi A, Villacis D, Yalamanchili R, Hatch GF 3rd. A comparison of rehabilitation methods after arthroscopic rotator cuff repair: a systematic review. Sports Health. 2015;7:326-34.

84. Kluczynski MA, Isenburg MM, Marzo JM, Bisson LJ. Does early versus delayed active range of motion affect rotator cuff healing after surgical repair? A systematic review and meta-analysis. Am J Sports Med. 2016;44:785-91.

85. Houck DA, Kraeutler MJ, Schuette HB, McCarty EC, Bravman JT. Early versus delayed motion after rotator cuff repair: a systematic review of overlapping meta-analyses. Am J Sports Med. 2017:45:2911-5.

86. Saltzman BM, Zuke WA, Go B, Mascarenhas R, Verma NN, Cole BJ, Romeo $A A$, Forsythe $B$. Does early motion lead to a higher failure rate or better outcomes after arthroscopic rotator cuff repair? A systematic review of overlapping meta-analyses. J Shoulder Elb Surg. 2017;26:1681-91.

87. Mazuquin BF, Wright AC, Russell S, Monga P, Selfe J, Richards J. Effectiveness of early compared with conservative rehabilitation for patients having rotator cuff repair surgery: an overview of systematic reviews. Br J Sports Med. 2018;52:111-21.

Ready to submit your research? Choose BMC and benefit from:

- fast, convenient online submission

- thorough peer review by experienced researchers in your field

- rapid publication on acceptance

- support for research data, including large and complex data types

- gold Open Access which fosters wider collaboration and increased citations

- maximum visibility for your research: over $100 \mathrm{M}$ website views per year

At $\mathrm{BMC}$, research is always in progress.

Learn more biomedcentral.com/submissions 